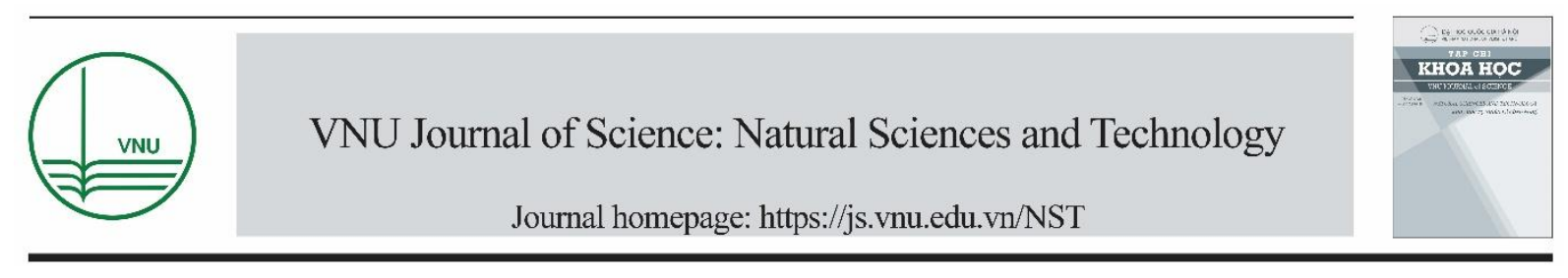

Original Article

\title{
Determination of 10-Hydroxy-2-Decenoic Acid Content - a Marker in Several Commercial Royal Jelly Products Collected in Vietnam
}

\author{
Duong Hong Anh*, Vu Minh Tuan, Van Thi Thanh Huyen, \\ Nguyen Manh Huy, Nguyen Thanh Dam \\ VNU Key Laboratory of Analytical Technology for Environmental Quality \& Food Safety Control, \\ VNU University of Science, 334 Nguyen Trai, Hanoi, Vietnam \\ Research Centre for Environmental Technology and Sustainable Development, VNU University of Science, \\ 334 Nguyen Trai, Hanoi, Vietnam
}

Received 24 October 2019

Revised 27 November 2019; Accepted 29 November 2019

\begin{abstract}
The optimized capillary electrophoresis (CE) was applied to separate and detect the 10 -hydroxy-2-decenoic acid (10-HAD) in royal jelly products. The method only requires that the sample solution need to be centrifuged and filtered before analyzed by the home-made capillary electrophoresis system. Firstly, 10-HDA was separated in a fused silica column with a diameter of 50 um using $20 \mathrm{mM}$ Tris/Acetic buffer ( $\mathrm{pH} \mathrm{8.5)}$ as a background electrolyte and a separation voltage of $-17 \mathrm{kV}$. Then, $10-\mathrm{HDA}$ was detected by capacitively coupled contactless conductivity detection $\left(C^{4} \mathrm{D}\right)$ with the migration time less than 8 minutes. Nine commercial products of royal jelly with Vietnamese and imported origin including pure royal jelly cream, lyophilized royal jelly (powder and gel) and honey with royal jelly were collected for analysis. The results of this study showed that content of 10 -HDA were detected in the range of $0.5 \mathrm{mg} / \mathrm{g}$ to $23.1 \mathrm{mg} / \mathrm{g}$. Using paired t test showed that the difference between results obtained from CE$\mathrm{C}^{4} \mathrm{D}$ method and from HPLC method as a reference method was not statistically significant.
\end{abstract}

Keywords: 10-HDA, royal jelly products, CE-C ${ }^{4} \mathrm{D}$.

\footnotetext{
* Corresponding author.

Email address: hoanggianga0@gmail.com

https://doi.org/10.25073/2588-1140/vnunst.4964
} 


\title{
VNU Journal of Science: Natural Sciences and Technology
} Journal homepage: https://js.vnu.edu.vn/NST

\section{Xác định hàm lượng axit 10-hydroxy-2-decenoic - "dấu chuẩn” trong một số sản phẩm sữa ong chúa thương mại thu thập tại Việt Nam}

\author{
Dương Hồng Anh*, Vũ Minh Tuấn, Văn Thị Thanh Huyền, \\ Nguyễn Mạnh Huy, Nguyễn Thanh Đàm \\ Phòng thí nghiệm Trọng điểm Công nghệ Phân tích phục vu kiểm định môi trường và an toàn thực phẩm, \\ Truờng Đại học Khoa học Tự nhiên, ĐHQGHN, 334 Nguyễn Trãi, Hà Nội, Việt Nam \\ Trung tâm Nghiên cưu Công nghệ môi trường và Phát triển bền vũng (CETASD), \\ Truờng Đại học Khoa học Tụ nhiên, ĐHQGHN, 334 Nguyễn Trãi, Hà Nội, Việt Nam
}

Nhận ngày 24 tháng 10 năm 2019

Chỉnh sửa ngày 27 tháng 11 năm 2019; Chấp nhận đăng ngày 29 tháng 11 năm 2019

\begin{abstract}
Tóm tắt: Hàm lượng axit 10-hydroxy-2-decenoic (10-HAD) trong các sản phẩm sữa ong chúa đã được phân tích bằng phương pháp điện di mao quản sử dụng detector độ dẫn không tiếp xúc. Phương pháp này chỉ đòi hỏi lọc dịch hòa tan mẫu đã ly tâm và sau đó thực hiện phân tích điện di mao quản. 10-HDA được phân tách trong cột fused sillica đường kính trong $50 \mu \mathrm{m}$ với dung dịch đệm điện ly là Tris/axetic $(20 \mathrm{mM}, \mathrm{pH}=8,5)$ và phát hiện bằng detector độ dẫn không tiếp xúc với thời gian di chuyển nhỏ hơn 8 phút. Chín mẫu sản phẩm chứa sữa ong chúa của Việt Nam và hàng nhập khẩu bao gồm sữa ong chúa tươi, sữa ong chúa đông khô (dạng bột và gel), mật ong sữa chúa đã được thu thập và phân tích. Hàm lượng $10-\mathrm{HDA}$ phát hiện được trong các sản phẩm nói trên ở khoảng 0,5 $\mathrm{mg} / \mathrm{g}$ tới $23,1 \mathrm{mg} / \mathrm{g}$. Sử dụng chuẩn $\mathrm{t}$ cho thấy các kết quả này được xác nhận là sai khác không có ý nghĩa khi so sánh với kết quả phân tích đối chứng bằng phương pháp sắc kí lỏng hiệu năng cao.
\end{abstract}

Tù khóa: $10-\mathrm{HDA}$, sản phẩm chứa sữa ong chúa, $\mathrm{CE}-\mathrm{C}^{4} \mathrm{D}$.

\section{Giới thiệu}

Axít 10 - Hydroxy - 2 - Decenoic (viết tắt: 10-HDA) còn được gọi là axít sữa ong chúa, là axit béo chưa bão hòa có hàm lượng cao nhất trong sữa ong chúa (thường chiếm khoảng 1,5 2,0\% trọng lượng). 10-HDA có các hoạt tính sinh học đặc hiệu đã được kiểm chứng bằng thực nghiệm như khả năng ngăn ngừa ung thư, ngăn ngừa lão hóa làn da, tăng cường khả năng miễn dịch và chống lại một loạt vi khuẩn, ... Do chỉ tồn tại trong sữa ong chúa tự nhiên, chứ không có trong các sản phẩm khác của ong như mật ong, phấn hoa, sáp ong..., nên 10-HDA được coi như một "dấu chuẩn"- marker của sữa ong chúa $[1,2]$.

\footnotetext{
* Tác giả liên hệ.

Địa chi email: hoanggianga0@gmail.com

https://doi.org/10.25073/2588-1140/vnunst.4964
} 
Các sản phẩm chứa sữa ong chúa hiện nay như sữa ong chúa tươi, sữa ong chúa đông khô, mật ong sữa chúa ... thường ít ghi nhãn về hàm lượng 10-HDA mà chỉ ghi tỷ lệ thành phần sữa ong chúa. Một số quốc gia như Thụy sỹ, Thổ Nhĩ Kỳ, Nhật Bản, Hàn Quốc, Ân độ, Trung Quốc đã có quy định về hàm lượng tối thiểu của 10-HDA trong sữa ong chúa tươi là $1,4 \%$ [3], với Brazil giá trị quy định này trong sữa ong chúa tươi là $2,0 \%$, và trong sữa ong chúa đông khô là $5,0 \%$ [4], Thái Lan và Thổ Nhĩ Kỳ có quy định đối với các sản phẩm khác chứa sữa ong chúa với hàm lượng 10-HAD tối thiểu là $0,16 \%$ [5]. Việc xác định hàm lượng thành phần này trong sữa ong chúa được thực hiện tại các phòng thí nghiệm bằng phương pháp sắc kí lỏng hiệu năng cao (HPLC) hoặc phương pháp sắc kí khí (GC). Trước khi phân tích bằng $\mathrm{GC}$ với detector khối phổ cần có bước tách chất phân tích ra khỏi nền mẫu, sau đó dẫn xuất hóa chúng thành các cấu tử dễ bay hơi qua phản ứng ankyl hóa, sylyl hóa [6]. Phương pháp HPLC với detector đo quang ở bước sóng $215 \mathrm{~nm}$ hoặc detector khúc xạ kế (RID) được sử dụng phổ biến hơn để xác định 10-HDA trong sữa ong chúa [5, 7-11]. Ngoài ra có một số ít nghiên cứu đã sử dụng phương pháp điện di mao quản với detector quang để phân tích 10-HAD [11, 12].

Bài báo này trình bày việc sử dụng phương pháp điện di mao quản với detector độ dẫn không tiếp xúc như một kỹ thuật phân tích đơn giản, yêu cầu chi phí thấp cho việc tách chiết và phân tích công cụ để xác định 10-HDA trong các sản phẩm sữa ong chúa. Một số mẫu thương mại như sữa ong chúa tươi, sữa ong chúa đông khô, mật ong sữa chúa đang bán trên thị trường Việt Nam đã được thu thập và phân tích bằng phương pháp nói trên.

\section{Thực nghiệm}

\subsection{Hóa chất, thiết bị}

Các hóa chất được sử dụng bao gồm: chất chuẩn 10-HDA 1g- Lot. RJQQM-IJ > 97\% từ Tokyo Chemical Industry (Nhật Bản), tris (hydroxymethyl) aminomethane (Tris) $\geq 99,8 \%$, axit axetic glacia (Ace) $\geq 99,8 \%$ từ Sigma-
Aldrich, metanol cho sắc ký lỏng Merck, nước cho sắc ký lỏng Merck, axit orthophotphoric 98\% Merck, $\mathrm{NaOH}$ dạng viên $\geq 98 \%$ Merck. Nước deion được dùng để pha các dung dịch chuẩn và xử lý mẫu, lấy từ máy lọc nước Simplicity UV, Millipore (USA) với chất lượng nước đầu ra siêu tinh khiết loại 1 có độ cách điện tại $25^{\circ} \mathrm{C} 18,2 \mathrm{M} \Omega . \mathrm{cm}$, hàm lượng TOC < $5 \mathrm{ppb}$. Màng lọc nilon $0,2 \mu \mathrm{m}$ Whatman được sử dụng trong quá trình xử lý mẫu.

Dung dịch chuẩn gốc $(1000 \mathrm{mg} / \mathrm{l})$ của 10 HDA được pha bằng cách cân chính xác $0,05 \mathrm{~g}$ 10-HDA, chuyển vào bình định mức $50 \mathrm{~mL}$, thêm nước deion đến vạch và lắc kĩ. Dung dịch chuẩn gốc này bền trong vòng 6 tháng nếu bảo quản ở $4^{\circ} \mathrm{C}$. Dung dịch chuẩn gốc được sử dụng để pha dung dịch các dung dịch lập đường chuẩn (5 tới $100 \mathrm{mg} / \mathrm{l}$ ) trên cơ sở pha loãng bằng nước deion với tỷ lệ thích hợp, các dung dịch chuẩn này bền trong vòng 1 tháng nếu bảo quản ở $4^{\circ} \mathrm{C}$. Dung dịch đệm điện ly (BGE) là Tris/axetic 20 $\mathrm{mM}$ pH 8,5 được chuẩn bị như sau: cân chính xác $0,242 \mathrm{~g}$ Tris chuyển vào bình định mức 100 $\mathrm{mL}$, thêm nước deion đến vạch và lắc kĩ, chuyển ra cốc sử dụng axit axetic băng chỉnh $\mathrm{pH}$ tới 8,5 , dung dịch BGE sử dụng trong ngày. Metanol, nước cho sắc ký lỏng và axit photphoric được sử dụng để chuẩn bị pha động cho sắc ký lỏng. Hỗn hợp metanol/ nước được pha với tỷ lệ $55: 45$ về thể tích, được điều chỉnh đến $\mathrm{pH} 2,5$ bằng axit photphoric, trước khi sử dụng cần siêu âm.

Tất cả các thí nghiệm được thực hiện trên hệ thiết bị điện di mao quản thao tác bằng tay $(\mathrm{CE})$ sử dụng detector đo độ dẫn không tiếp xúc $\left(\mathrm{C}^{4} \mathrm{D}\right)$ tại Phòng thí nghiệm trọng điểm công nghệ phân tích phục vụ kiểm định môi trường và an toàn thực phẩm (KLATEFOS), Trường Đại học Khoa học Tự Nhiên, Đại học Quốc gia Hà Nội. Hệ thiết bị sử dụng nguồn cao thế $\pm 25 \mathrm{kV}$ (Spellman, Anh), ghi dữ liệu nhờ bộ ghi e-corder (eDAQ, Úc). Cột mao quản silica nóng chảy đường kính trong $50 \mu \mathrm{m}$, đường kính ngoài là $365 \mu \mathrm{m}$ với độ dài tổng $\left(\mathrm{L}_{\text {tot }}\right) 60 \mathrm{~cm}$ và độ dài hiệu dụng $\left(\mathrm{L}_{\text {eff }}\right)$ $48 \mathrm{~cm}$ (Agilent, Mỹ) được sử dụng để tách chất. Trước lần phân tích đầu tiên của mỗi ngày, mao quản được ổn định hóa bằng dung dịch $\mathrm{NaOH}$ 
$0,1 \mathrm{M}$ trong 10 phút, sau đó là nước deion trong 10 phút và $B G E$ trong 30 phút. Sau mỗi phép đo, mao quản được rửa bằng $\mathrm{BGE}$ trong vòng 3 phút.

Phân tích so sánh được thực hiện trên cơ sở phương pháp tham khảo tại tài liệu [7], sử dụng thiết bị sắc ký lỏng hiệu năng cao LC $20 \mathrm{AB}$ Shimadzu với detector mảng diod (DAD), cột phân tích RP-C18 $25 \mathrm{~cm}$ x 4,6 mm x $5 \mu \mathrm{m}$, pha động sử dụng là hỗn hợp metanol/ nước tỷ lệ 55:45 về thể tích thêm axit photphoric tới $\mathrm{pH} 2,5$, tốc độ dòng pha động $1 \mathrm{ml}$ theo chế độ đẳng dòng, 10-HDA được nhận biết tại bước sóng hấp thụ $215 \mathrm{~nm}$.

\subsection{Phân tích 10-HAD trong mẫu thực}

Chín mẫu sản phẩm chứa sữa ong chúa (cả sản xuất trong nước và nhập ngoại) bao gồm: sữa ong chúa tươi, sữa ong chúa đông khô dưới dạng bột rắn hoặc gel, mật ong có trộn lẫn sữa ong chúa tươi đã được mua trên thị trường.

Quy trình phân tích 10-HDA trong các sản phẩm bằng kỹ thuật điện di mao quản đã được nhóm nghiên cứu tối ưu hóa các thông số: thành phần, nồng độ, $\mathrm{pH}$ của $\mathrm{BGE}$, điện thế tách, thời gian bơm mẫu, dung môi hòa tan mẫu, loại màng lọc. Trong bài báo này không đưa kết quả các khảo sát xây dựng phương pháp phân tích, chỉ áp dụng các điều kiện đã tối ưu hóa để đánh giá phương pháp và áp dụng cho phân tích mẫu thực.

Quy trình phân tích cụ thể được thực hiện như sau: cân chính xác một lượng mẫu (khoảng $1 \mathrm{~g}$ ) hòa mẫu vào dung môi metanol/nước deion (tỷ lệ 30:70 về thể tích), định mức tới $50 \mathrm{ml}$, lắc, ly tâm, lọc qua màng lọc nilon $0,2 \mu \mathrm{m}$ trước khi bơm vào thiết bị $\mathrm{CE}-\mathrm{C}^{4} \mathrm{D}$. Việc phân tích 10 HDA trên hệ thiết bị CE-C ${ }^{4} D$ được thực hiện với các điều kiện: dung dịch BGE Tris/axetic $20 \mathrm{mM}$ $\mathrm{pH} 8,5$, điện thế tách $-17 \mathrm{kV}$, thời gian bơm mẫu $30 \mathrm{~s}$. Thời gian di chuyển để định tính 10-HDA là $430 \pm 1(\mathrm{~s})$. Để hạn chế ảnh hưởng nền tới việc định tính chất phân tích tín hiệu trong mẫu bơm được khẳng định lại sau khi thêm chuẩn vào dịch lọc. Đường chuẩn được xây dựng theo phương pháp ngoại chuẩn ( 5 điểm) với nồng độ $10-\mathrm{HDA}$ lần lượt là $5,10,20,50$ và $100 \mathrm{mg} / \mathrm{l}$. Việc định lượng được thực hiện bằng phương pháp đường chuẩn, mẫu được bơm lặp 3 lần lấy kết quả trung bình.

Từ kết quả phân tích dịch bơm vào $\mathrm{CE}-\mathrm{C}^{4} \mathrm{D}$ có thể tính được nồng độ 10-HDA trong dịch lọc hòa tan mẫu tùy theo việc có pha loãng dịch lọc này trước khi bơm hay không. Tiếp theo từ nồng độ 10-HDA trong dịch lọc và các hệ số khi xử lý mẫu có thể tính được hàm hượng 10-HDA có trong sản phẩm ban đầu theo công thức như sau:

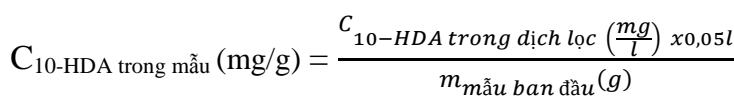

\section{3. Đảm bảo và kiểm soát chất lượng phân tích}

Giá trị IDL (giới hạn phát hiện của thiết bị) được tính bằng nồng độ chất phân tích trong dung môi cho tín hiệu gấp 3 lần nhiễu nền. Giá trị giới hạn phát hiện, giới hạn định lượng của phương pháp (MDL, MQL) của 10-HDA được tính từ giá trị IDL và các hệ số quy đổi tương ứng với hòa tan $1,0 \mathrm{~g}$ mẫu trong $50 \mathrm{ml}$ dung môi.

Trong nghiên cứu này, độ lặp lại của phương pháp được xác định thông qua giá trị độ lệch chuẩn tương đối (RSD) của thời gian di chuyển và diện tích pic khi thực hiện 7 phép phân tích lặp lại đối với các dung dịch chuẩn có nồng độ 10-HDA là $20 \mathrm{mg} / \mathrm{l}$, độ tái lặp được tính bằng giá trị độ lệch chuẩn tương đối (RSD) của các phép phân tích lặp lại trong 8 ngày liên tiếp cũng với mức nồng độ $20 \mathrm{mg} / \mathrm{l}$. Độ chính xác được xác định bằng hiệu suất thu hồi của mẫu thêm chuẩn vào nền sữa ong chúa tươi với mức nồng độ thêm tương đương $20 \mathrm{mg} / \mathrm{l} 10$-HAD trong dịch bơm và mẫu thêm chuẩn vào nền sữa ong chúa đông khô dạng gel với mức nồng độ thêm tương đương $5 \mathrm{mg} / \mathrm{l} 10-\mathrm{HAD}$ trong dịch bơm.

Các kết quả phân tích bằng $\mathrm{CE}-\mathrm{C}^{4} \mathrm{D}$ được tính trung bình dựa trên 3 lần bơm mẫu lặp. 6/9 mẫu được phân tích so sánh bằng phương pháp HPLC sử dụng detector DAD.

\section{Kết quả và thảo luận}

\section{1. Đánh giá phương pháp}

Kết quả đánh giá phương pháp phân tích đã xây dựng được trình bày trong bảng 1 . Hệ số hồi 
quy tuyến tính của đường chuẩn thu được có giá tri tốt với $\mathrm{R}^{2}>0,999$. Hiệu suất thu hồi đạt từ 97 đến $106 \%$ khi thêm chuẩn 10 -HDA vào các nền mẫu thực. Giá trị giới hạn định lượng của phương pháp thu được là $0,13 \mathrm{mg} 10-\mathrm{HDA} / \mathrm{g}$ sản phẩm sữa ong chúa. Giá trị này phù hợp để xác định 10-HDA trong sữa ong chúa tươi nguyên chất, cũng như trong những sản phẩm chứa sữa ong chúa với tỷ lệ pha trộn thông thường cỡ 10 $5 \%$. Độ lặp lại trong ngày của diện tích pic và thời gian di chuyển được thể hiện dưới dạng độ lệch chuẩn tương đối (RSD) trên nền nước deion đều nhỏ hơn 3\% khi phân tích lặp lại 7 lần. Độ tái lặp khi thực hiện phép phân tích trong liên tục

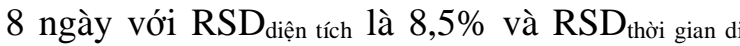
chuyển là $3,2 \%$. Các giá trị của độ lặp lại và độ tái lặp đối với diện tích pic và thời gian di chuyển và cho thấy phương pháp phân tích là lặp lại và có độ ổn định tốt.

Bảng 1. Các thông số đánh giá phương pháp CE-C ${ }^{4} \mathrm{D}$ phân tích $10-\mathrm{HAD}$ trong sản phẩm sữa ong chúa

\begin{tabular}{|c|c|}
\hline & 10-HDA \\
\hline Khoảng đường chuẩn (mg/l) & $5 \div 100$ \\
\hline Hệ số tương quan $\mathrm{R}^{2}$ & 0,9995 \\
\hline Thời gian di chuyển (s) (n=7) & $430 \pm 1$ \\
\hline $\mathrm{IDL} *(\mathrm{mg} / \mathrm{l})$ & 1,24 \\
\hline MDL**(mg/g sản phẩm sữa ong chúa) & 0,039 \\
\hline MQL**(mg/g sản phẩm sữa ong chúa) & 0,13 \\
\hline \multirow[t]{2}{*}{ Độ lặp lại: $\mathrm{RS}$} & 2,24 \\
\hline & 0,26 \\
\hline Độ tái lặp: RSD diện tích $(\%)$ (8 ngày) & 8,48 \\
\hline $\mathrm{RSD}_{\text {thời gian di chuyển }}(\%)$ (8 ngày) & 3,18 \\
\hline Hiệu suất thu hồi (\%) trên các nền mẫu thực & $97 \div 105$ \\
\hline $\begin{array}{l}\text { * Theo tỷ lệ tín hiệu/nhiễu: } S / N= \\
\text { ** Tính dụa trên giá trị IDL và }\end{array}$ & $\tilde{\tilde{n}}$ \\
\hline
\end{tabular}

Hình 1 là điện di đồ của mẫu chuẩn, một mẫu thực (mẫu 6 - sữa ong chúa đông khô dạng gel) và mẫu thêm chuẩn (để khẳng định định tính tín hiệu 10-HDA khi phân tích bằng phương pháp CE-C ${ }^{4} \mathrm{D}$ nói trên).

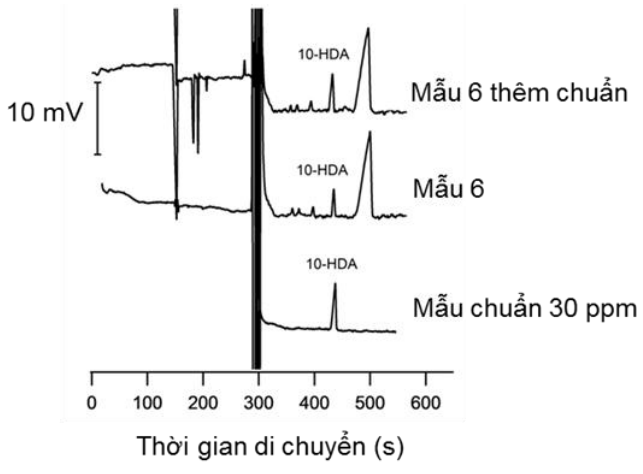

Hình 1. Điện di đồ phân tích 10-HDA trong mẫu chuẩn, mẫu thực và mẫu thêm chuẩn

Các điều kiện điện: di thế tách $-17 \mathrm{kV}$; thời gian bơm mẫu $30 \mathrm{~s}$; mao quản silica nóng chảy I.D $=50 \mu \mathrm{m}, \mathrm{L}_{\mathrm{tot}}=60 \mathrm{~cm}, \mathrm{~L}_{\text {eff }}=48 \mathrm{~cm}$.

\subsection{Kết quả phân tích các mẫu thực}

Sau khi xây dựng đường chuẩn và đánh giá phương pháp, 9 mẫu thật (bao gồm 3 mẫu sữa ong chúa tươi, 1 mẫu viên nang sữa ong chúa đông khô dạng bột, 3 mẫu viên nang sữa ong chúa đông khô dạng gel, 2 mẫu mật ong sữa chúa) đã được phân tích nhằm định lượng 10HDA trong thành phần. Giản đồ điện di phân tích một số mẫu được minh họa trên hình 2.

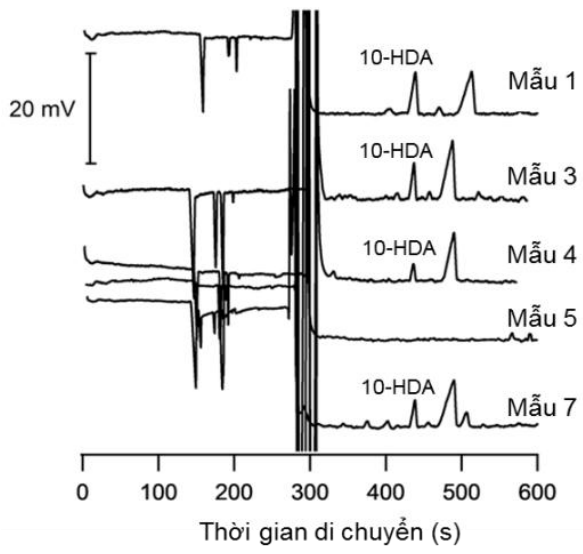

Hình 2. Điện di đồ phân tích 10-HDA trong một số mẫu sản phẩm sữa ong chúa; mẫu 1: sữa ong chúa tươi, mẫu 3: sữa ong chúa/ấu trùng

Các điều kiện điện: di thế tách $-17 \mathrm{kV}$; thời gian bơm mẫu $30 \mathrm{~s}$; mao quản silica nóng chảy $\mathrm{I} . \mathrm{D}=50 \mu \mathrm{m}, \mathrm{L}_{\mathrm{tot}}=60 \mathrm{~cm}, \mathrm{~L}_{\mathrm{eff}}=48 \mathrm{~cm}$. 
Bảng 3. Kết quả phân tích hàm lượng 10-HDA trong sản phẩm sữa ong chúa

\begin{tabular}{|c|c|c|c|c|c|}
\hline \multirow[t]{2}{*}{ Loại mẫu/xuất xứ } & \multirow[t]{2}{*}{ Ghi nhãn } & \multirow[t]{2}{*}{ Code mẫu } & \multicolumn{3}{|c|}{ Hàm lượng 10-HDA (mg/g) } \\
\hline & & & $C E-C^{4} D(n=3)$ & HPLC & Chênh lệch (\%) \\
\hline \multirow{3}{*}{$\begin{array}{l}\text { Sữa ong chúa } \\
\text { tươi/Việt Nam }\end{array}$} & $100 \%$ sữa ong chúa & Mẫu 1 & $21,38 \pm 0,89$ & 21,52 & $-0,7$ \\
\hline & $100 \%$ sữa ong chúa & Mẫu 2 & $9,14 \pm 0,27$ & - & \\
\hline & $100 \%$ sữa ong chúa & Mẫu 9 & $23,07 \pm 0,99$ & 22,83 & $+1,0$ \\
\hline $\begin{array}{l}\text { Viên nang sữa ong } \\
\text { chúa đông khô } \\
\text { dạng bột/ Việt Nam }\end{array}$ & $\begin{array}{l}450 \mathrm{mg} \text { sữa ong chúa } \\
\text { và ấu trùng ong chúa } \\
\text { đông khô, } 50 \mathrm{mg} \text { tá } \\
\text { dược }\end{array}$ & Mẫu 3 & $13,20 \pm 0,30$ & 13,77 & $-4,1$ \\
\hline \multirow[t]{3}{*}{$\begin{array}{l}\text { Viên nang sữa ong } \\
\text { chúa đông khô } \\
\text { dạng gel/Úc, Mỹ }\end{array}$} & $\begin{array}{l}\text { 183,3 mg sữa ong } \\
\text { chúa đông khô }(3: 1) \\
6 \% \text { 10-HAD tương } \\
\text { đương } 11 \mathrm{mg} \text { 10-HDA }\end{array}$ & Mẫu 4 & $0,54 \pm 0,01$ & - & \\
\hline & $6 \%$ 10-HDA & Mẫu 5 & $<0,13$ & $<0,036$ & \\
\hline & $\begin{array}{l}500 \mathrm{mg} \text { (từ } 152 \mathrm{mg} \\
\text { sữa ong chúa cô đặc, } \\
\text { tương đương } 500 \mathrm{mg} \\
\text { sữa ong chúa tươi) }\end{array}$ & Mẫu 6 & $2,58 \pm 0,07$ & 2,60 & $-0,7$ \\
\hline \multirow[t]{2}{*}{$\begin{array}{l}\text { Mật ong sữa chúa/ } \\
\text { Việt Nam }\end{array}$} & $\begin{array}{l}10 \% \text { sữa chúa tươi, } \\
90 \% \text { mật ong hoa rừng }\end{array}$ & Mẫu 7 & $1,72 \pm 0,04$ & 1,65 & $+4,2$ \\
\hline & $\begin{array}{l}10 \% \text { sữa chúa tươi, } \\
90 \% \text { mật ong }\end{array}$ & Mẫu 8 & $<0,13$ & - & \\
\hline \multirow[t]{2}{*}{ Loại mẫu/xuất xứ } & Ghi nhãn & Code mẫu & \multicolumn{3}{|c|}{ Hàm lượng 10-HDA (mg/g) } \\
\hline & & & CE-C ${ }^{4} D(n=3)$ & HPLC & Chênh lệch (\%) \\
\hline MQL (mg/g) & & & 0,13 & 0,036 & $\begin{aligned} \mathrm{t}_{\text {tính }} & =0,551 \\
\mathrm{t}_{\text {bảng }}(\mathrm{p}=95 \%) & =2,776\end{aligned}$ \\
\hline
\end{tabular}

Ghi chú: - : không phân tích

Độ chênh lệch $=\left\{\left(\mathrm{C}_{\mathrm{CE}-\mathrm{C} 4 \mathrm{D}}-\mathrm{C}_{\mathrm{HPLC}}\right) / \mathrm{C}_{\mathrm{HPLC}}\right\} \times 100 \%$

Các kết quả phân tích mẫu thực thu được trong bảng 3 cho thấy hàm lượng 10-HDA phát hiện được trong khoảng $9,1-23,1 \mathrm{mg} / \mathrm{g}$ đối với sữa ong chúa tươi, các giá trị này là tương đối phù hợp với khoảng giá trị thông thường từ $1,5-$ $2 \%$ và tiêu chuẩn một số quốc gia quy định là $1,4 \%$. Trong các mẫu viên nang sữa ong chúa đông khô dạng gel nhập khẩu mặc dù ghi nhãn hầu hết là sữa ong chúa đông khô theo tỷ lệ 1:3, tức là hàm lượng 10-HDA sẽ cao hơn trong sữa ong chúa tươi cỡ 3 lần, tương đương khoảng 60 $\mathrm{mg} / \mathrm{g}$ nhưng trên thực tế khi phân tích hàm lượng 10-HDA chỉ có $0,5-2,6 \mathrm{mg} / \mathrm{g}$. Cá biệt mẫu số 5 không phát hiện thấy 10-HDA (cả bằng phương pháp HPLC) cũng như không phát hiện thấy thành phần amino axit tự do khi phân tích (không trình bày kết quả ở đây) cho thấy đây có thể là sản phẩm giả mạo (sản phẩm này được mua qua shop online). Trong mật ong sữa chúa, mặc dù thành phần sữa ong chúa ghi nhãn là $10 \%$ nhưng chỉ một mẫu tìm thấy $10-H D A$ còn một mẫu không phát hiện (mẫu này có phát hiện thấy amino axit tự do).

So sánh các kết quả phân tích thu được khi dùng phương pháp $\mathrm{CE}-\mathrm{C}^{4} \mathrm{D}$ và phương pháp truyền thống HPLC sử dụng chuẩn $\mathrm{t}$ cho thấy: $\mathrm{t}_{\text {tính }}=0,551, \mathrm{t}_{\text {bảng }}(\mathrm{p}=95 \%)=2,776$, giá trị $\mathrm{t}_{\text {tính }}<$ tbảng khẳng định sai khác giữa kết quả thu được từ hai phương pháp phân tích (theo từng cặp) là không có nghĩa hay nói cách khác các kết quả thu được từ phương pháp $C E-C^{4} D$ và $\mathrm{HPLC}$ là tương đương. Kết quả này đã chứng minh được độ tin cậy khi sử dụng phương pháp $\mathrm{CE}-\mathrm{C}^{4} \mathrm{D}$ cho đối tượng 10-HDA. 
Bảng 4 cung cấp kết quả nồng độ 10-HDA xác định được trong các sản phẩm sữa ong chúa tại một số quốc gia như Ý, Rumani, Thổ Nhĩ Kỳ, Chi lê, Trung Quốc và trong nghiên cứu này. Các kết quả cho thấy sự có mặt của 10-HDA trong các sản phẩm sữa ong chúa tươi có nguồn gốc ở nhiều quốc gia là tương tự nhau. Tuy nhiên hàm lượng của hợp chất này trong các sản phẩm đã chế biến như sữa ong chúa đông khô, mật ong sữa chúa, sâm sữa chúa...có khoảng dao dộng khá cao, có thể phụ thuộc vào tỷ lệ pha sữa ong chúa, vào chất lượng nguồn sữa ong chúa cũng như sự thay đổi của hợp chất này theo thời gian sau khi pha trộn.

Bảng 4. Hàm lượng 10-HDA trong sản phẩm sữa ong chúa của một số quốc gia

\begin{tabular}{llcl}
\hline Loại mẫu & Xuất xứ & Hàm lượng & Tài liệu tham khảo \\
& & $0,8-3,2$ & {$[11]$} \\
Sữa ong chúa tươi & Italia $(\mathrm{n}=8)$ & $1,2-1,9$ & {$[11]$} \\
& Châu Âu $(\mathrm{n}=6)$ & $1,41-3,08$ & {$[8]$} \\
& Rumani - địa phương $(\mathrm{n}=19)$ & $1,54-2,08$ & {$[8]$} \\
& Rumani - thương mại $(\mathrm{n}=9)$ & $0,33-2,54$ & {$[9]$} \\
& Thổ Nhĩ Kỳ $(\mathrm{n}=10)$ & $0,019-0,034$ & {$[12]$} \\
& Chi lê $(\mathrm{n}=2)$ & $1,85-2,15$ & {$[7]$} \\
& Mỹ - địa phương $(\mathrm{n}=3)$ & $1,26-2,21$ & {$[9]$} \\
& Trung quốc $(\mathrm{n}=20)$ & $0,9-2,3$ & Nghiên cứu này \\
\hline Sữa ong chúa đông khô & Việt Nam $(\mathrm{n}=3)$ & 0,019 & {$[12]$} \\
& Chi lê $(\mathrm{n}=2)$ & $3,01-6,19$ & {$[10]$} \\
& Trung Quốc $(\mathrm{n}=10)$ & 1,32 & Nghiên cứu này \\
& Việt Nam $(\mathrm{n}=1)$ & $0,05-0,26$ & Nghiên cứu này \\
\hline Sản phẩm khác chứa sữa & Úc, Mỹ $(\mathrm{n}=2)$ & nd $-0,054$ & {$[8]$} \\
ong chúa & Thố Nhĩ Kỳ $(\mathrm{n}=7)$ & $0,43-6,28$ & {$[7]$} \\
& Mỹ - thương mại $(\mathrm{n}=11)$ & nd $-0,98$ & {$[10]$} \\
& Trung Quốc $(\mathrm{n}=30)$ & nd $-0,17$ & Nghiên cứu này \\
\hline
\end{tabular}

\section{Kết luận}

Phương pháp điện di mao quản sử dụng detector độ dẫn không tiếp xúc để phân tích hàm lượng axit 10-hydroxy-2-decenoic - dấu chuẩn trong các sản phẩm sữa ong chúa là một kỹ thuật đơn giản, hiệu quả. Quá trình xử lý mẫu chỉ đòi hỏi ly tâm, lọc dịch hòa tan mẫu và phân tích điện di trực tiếp với thời gian phát hiện nhỏ chất phân tích nhỏ hơn 8 phút. Với giới hạn phát hiện và định lượng đã xác định và khoảng hàm lượng thông thường của 10-HDA trong sữa ong chúa tươi nguyên chất, phương pháp này cho phép phát hiện 10-HDA trong những sản phẩm chứa sữa ong chúa với tỷ lệ pha trộn cỡ $2 \%$ sữa ong chúa. Với sai khác không có ý nghĩa thống kế khi so sánh với kết quả phân tích bằng HPLC, cùng sự tiện lợi về kỹ thuật, tiết kiệm về thời gian và chi phí cho thấy tính cạnh tranh của phương pháp điện di mao quản trong phân tích thực phẩm khi so sánh với kỹ thuật sắc ký truyền thống.

\section{Lò̀i cảm ơn}

Nghiên cứu được thực hiện trong khuôn khổ đề tài NCKH cấp Đại học Quốc gia Hà Nội mã số QG.18.05, các tác giả xin trân trọng cảm ơn.

\section{Tài liệu tham khảo}

[1] M. Viuda Martos, Y. Ruiz Navajas, J. Fernández López, J.A. Pérez Álvarez, Functional properties of honey, propolis, and royal jelly", Journal of food science 73 (2007) 117-124. https://doi:10.1111/j. 1750-3841.2008.00966.x.

[2] M.F. Ramadan, A. Al-Ghamdi, Bioactive compounds and health-promoting properties of 
royal jelly: A review, Journal of Functional Foods 4 (2012) 39- 52. https://doi.org/10.1016/j.jff. 2011. 12.007.

[3] Dimitrios Kanelis, Chrysoula Tananaki, Vasilis Liolios, Maria Dimou, Georgios Goras, Maria Anna Rodopoulou, Emmanuel Karazafris, and Andreas Thrasyvoulou, A suggestion for royal jelly specifcations, Archives of Industrial Hygiene and Toxicology 66 (2015) 275-284. Https://doi: 10.1515/aiht-2015-66-2651.

[4] Brazil Ministério Da Agricultura e Do Abaste Imento. Secretaria De Defesa Agropecuária. Instrução normativa $\mathrm{n}^{\circ} 3$, de 19 de janeiro de 2001. Regulamento técnico para fxação de identidade e qualidade de geléia real, Regulamento técnico para fxação de identidade e qualidade de geléia real liofilizada. Http:// www.engetecno.com.br/port/ legislacao/mel_geleia_real.htm.

[5] Mahmut Genc, Abdurrahman Aslan, Determination of trans-10-hydroxy-2-decenoic acid content in pure royal jelly and royal jelly products by column liquid chromatography, Journal of Chromatography A. 839 (1999) 265 268. Http://doi: 10.1016/s0021-9673(99)00151-x.

[6] F. Ferioli, E. Armaforte, M.F. Caboni, Comparison of the Lipid Content, Fatty Acid Profile and Sterol Composition in Local Italian and Commercial Royal Jelly Samples" Journal of the American Oil Chemists' Society, 91(2014) 875-884. Http://doi. org/10.1007/s11746-014-2446-x.

[7] J. Kim, J. Lee, Quantitative analysis of trans-10hydroxy-2-decenoic acid in royal jelly products purchased in USA by high performance liquid chromatography", Journal of Apicultural Science, 54 (2010) 77-85. Https://pdfs.semanticscholar. org/e85f/dfc1823d778a40c ca2eefa4be8ba4b4d98 6c.pdf.

[8] C.I. Pavel, L.A. Mărghitaş, D.S. Dezmirean, L.I. Tomoş, V. Bonta, A. Şapcaliu, A. Buttstedt, Comparison between local and commercial royal jelly-use of antioxidant activity and 10-hydroxy-2decenoic acid as quality parameter", Journal of Apicultural Research 53 (2014) 116-123. https:// doi.org/10.3896/IBRA.1.53.1.12.

[9] Mahmut Genc, Abdurrahman Aslan, Determination of trans-10-hydroxy-2-decenoic acid content in pure royal jelly and royal jelly products by column liquid chromatography, Journal of Chromatography A, 839 (1999) 265 268. Https://doi:10.1016/s0021-9673(99)00151-x.

[10] Jinhui Zhou, Xiaofeng Xue, Yi Li, Jinzhen Zhang and Jing Zhao, Optimized determination method for trans-10-hydroxy-2-decenoic acid content in royal jelly by high-performance liquid chromatography with an internal standard, Journal of AOAC International, 90(2007) 244-249. https://www.ncbi.nlm.nih.gov/pubmed/17373456.

[11] Federico Ferioli, Gian Luigi Marcazzan, Maria Fiorenza Caboni, Determination of (E)-10hydroxy-2-decenoic acid content in pure royal jelly: A comparison between a new CZE method and HPLC", J. Sep. Sci 30 (2007) 1061-1069. Http://doi:10.1002/jssc.200600416.

[12] Orlando Muñoz, Susana Decap, Francisco Ruiz, José Arbildua, Octavio Monasterio, Determination of 10- hydroxy-2-decenoic acid in royal jelly by capillary electrophoresis, J. Chil. Chem. Soc., 56 (2010) 738-740. http://dx.doi.org/10.4067/S0717 97072011000300004. 